\title{
IMPACT OF LOCAL INJECTION OF HUMAN GROWTH HORMONE VERSUS LOW-LEVEL LASER THERAPY ON MANDIBULAR CONDYLE IN ARTHRITIC GUINEA PIGS
}

\author{
Sami Abdulraqeb Al-Dubai*, Amira Mohsen Elsherbini**, \\ Rehab Risk El Zehary ${ }^{* * *}$ and Wael Mohamed Said Ahmed ${ }^{* * * *}$
}

\begin{abstract}
Objectives: This study aimed to compare the effect of intra-articular injection of human growth hormone (HGH) versus low-level laser therapy (LLLT) on mandibular condyle in arthritic guinea pigs.

Methods: A total of 30 male guinea pigs with average weight $(250 \mathrm{~g}-350 \mathrm{~g})$ had a temporomandibular joint (TMJ) arthritis induced by locally injecting complete freund's adjuvant (CFA) intra-articularly in the right side. Then they were divided into three groups; control, HGH and LLLT. The applications of both treatments were done every two days for two weeks, then all groups were euthanized. Arthritic TMJs were dissected and processed for histological, histomorphometric and statistical analysis..
\end{abstract}

Results: Histological evaluation showed increased thickness of articular cartilage and formation of new subchondral bone trabeculae and narrowing of marrow spaces in both study groups with statistical significant differences compared to control group.

Conclusion: Treatment of TMJ arthritis with HGH showed improvements in all parameters tested. HGH can be a promising modality to repair degenerative changes associated with CFA induced arthritis.

KEYWORDS: Temporomandibular joints, Arthritis, human growth hormone, low-level laser therapy.

\footnotetext{
* MDs of Oral Surgery, Faculty of Dentistry, Mansoura University, Mansoura, Egypt

** Lecturer of Oral Biology, Faculty of Dentistry, Mansoura University, Mansoura, Egypt

*** Assistant Professor of Oral Biology, Faculty of Dentistry, Mansoura University, Mansoura, Egypt.

**** Assistant Professor of Oral and Maxillofacial Surgery, Faculty of Dentistry, Mansoura University, Mansoura, Egypt.
} 


\section{INTRODUCTION}

Temporomandibular joint disorders refer to conditions that affect TMJs and masticatory muscles. Degenerative joint diseases (DJDs) are characterized by degeneration of the joint in addition to facial pain which result in disability. ${ }^{1}$ Rheumatoid arthritis (RA) is a systemic heterogeneous autoimmune disease. Among several animal models, ${ }^{2}$ guinea pigs are considered appropriate for studying various inflammatory and infectious diseases including arthritis. $^{3}$

The HGH is a pleiotropic hormone secreted by the pituitary gland. The effect of $\mathrm{HGH}$ is produced through intermediate substances called somatomedins. ${ }^{4}$ Although it is known primarily as potent stimulant and regulator in somatic growth, it is involved also in bone and muscle mass regulation. ${ }^{5,6} \mathrm{HGH}$ decreases the development of type I diabetes. ${ }^{4} \mathrm{HGH}$ involvement in immune system regulation is thought to be via modulating several aspects such as thymic development, B cell responses and antibody production, natural killer cell and macrophage activity. ${ }^{7}$

The LLLT has excelled in treatment of DJDs due to its anti-inflammatory, analgesic and regenerative effects. LLLT might possess bio-stimulator properties that lead to blockage of some proinflammatory mediators, for example interleukin 1 beta (IL-1 $\beta$ ), Cyclooxygenase-2 (COX-2), prostaglandins E2 (PGE2) and Tumor necrosis factor- alpha (TNF $\alpha){ }^{8}$ In addition to modulation of tissue repair, matrix metalloproteinases (MMPs) activity reduction and analgesic properties via direct irradiation without thermal response. ${ }^{9}$

There is no curative treatment of RA. ${ }^{10}$ The proposed treatment should at least reduce or even prevent joint destruction and deformity in order to restore the joint function. This study aimed to compare the effect of HGH and LLLT in treatment of RA.

\section{MATERIALS AND METHODS}

This study was approved by the ethical committee, Faculty of Dentistry, Mansoura University, Egypt (NO 09030718). The sample size was calculated using $\mathrm{G}^{*}$ Power 3.1.9.2. In a oneway ANOVA study, a sample sizes of 10 guinea pigs in each of the three groups (Control, HGH, LLLT) achieved $95 \%$ power to detect differences among the means versus the alternative of equal means using an F test (ANOVA: fixed effects, omnibus, one way) with a 0.05000 significance level to detect a hypothesized large effect size of .8. Thirty male guinea pigs with average weight $(250 \mathrm{~g}-350 \mathrm{~g})$, were acclimatized under standardized conditions; temperature, humidity and housed in individual with free access to food.

\section{Animals Grouping}

Arthritis was induced by single intra-articular injection into the right TMJ with $0.125 \mathrm{ml}$ of CFA (Sigma-Aldrich Chemie Gmbh, Germany) and arthritis development was evaluated by a blind examiner 3 times a week ${ }^{11}$

The exact position of needle insertion was established by palpation of the condyle, while the mandible was manually moved from side to side.

Arthritic animals were allocated randomly into 3 equal groups. The right TMJs were injected with normal saline in control group $(\boldsymbol{C G})$ and $\mathrm{HGH}$ (Somatropin 4 IU, Sedico Pharmaceutical Co., Egypt) in $\boldsymbol{H G H}$ group (HGHG), while exposed to LLLT (Quicklase, UK) in LLLT group (LLLTG). All animals were euthanized after 8 days from the last of saline, $\mathrm{HGH}$ or laser session via overdose of xylazine and ketamine.

\section{HGH injection}

After 28 days from induction of arthritis, anesthesia was induced by injection of ketamine $(75 \mathrm{mg} / \mathrm{kg}$ body weight) and xylazine $(25 \mathrm{mg} / \mathrm{kg}$ body weight) intra-peritoneally. TMJ area was shaved and disinfected with $10 \%$ povidone iodine, 
then $1 \mathrm{~mL}$ of $2 \mathrm{mg} / \mathrm{kg}$ body weight of $\mathrm{HGH}$ was injected in the right TMJ every 2 days for twelve days (6 injections). ${ }^{12}$ While CG was injected using the same protocol but with saline.

\section{LLLT}

GaAlAs laser with $1064 \mathrm{~nm} \lambda$, $6 \mathrm{~W}$ output power (Quicklase, UK) was used. Animals right TMJs received an application of $3 \mathrm{~J} / \mathrm{cm} 2$ at a single point, 12 seconds duration, seven sessions and forty eight hour intervals. ${ }^{9}$

\section{Histological \& statistical analysis}

TMJ sections were fixed, decalcified then processed and stained with routine $\mathrm{H} \& \mathrm{E}$ stain. Masson's trichrome and Toluidine blue stains were used for collagen and glycosaminoglycan detection respectively, followed by histomorphomrtric analysis.

Ten different standardized fields were analyzed, by a blind examiner, for each group. The percentage of collagen in newly formed subchondral bone, disk thickness, upper and lower compartments, marrow spaces and thickness of cartilage, represented by distance from the superior border of the mandibular cartilage to boundary with the zone of endochondral ossification, were measured, followed by statistical analysis.

Qualitative data were initially tested for normality using Shapiro-Wilk test (Statistical Package for Social Science software computer program version 23 (SPSS, Inc., Chicago, IL, USA). The data was described using mean and standard deviation. One way Analysis of variance (ANOVA) followed by Tukey were used for comparing data. $\mathrm{P}$ value less than 0.05 was considered statistically significant.

\section{RESULTS AND DISCUSSION}

TMJ represents unique properties compared to other joints, and is frequently affected by RA ${ }^{13}$, which leads to arthritic changes in all components of the joint, that may reach up to complete loss of the condyle and exposure of subchondral bone. CFA arthritic experimental model has been successfully used, and mandated in certain strain of animals as guinea pigs for arthritic induction. ${ }^{14}$

Clinically the injected TMJs showed signs of inflammation, but there was no weight loss in guinea pigs.

The CG revealed, in $\mathrm{H} \& \mathrm{E}$ stained sections, articular cartilage thinning, irregularities and severe atrophy in some parts. In addition to subchondral bone $(\mathrm{sb})$ destruction resulting in exposure of articular cartilaginous region with an increase in marrow spaces and osteoclastic activities represented by Hawship's lacunae Figure (Fig.1, A). These results were in agreement with those of Lemos et al. ${ }^{15} \mathrm{Xu}$ et al., ${ }^{16}$ who observed destructive changes in CFA-injected TMJ.

While $\mathrm{H} \& \mathrm{E}$ sections of HGHG, showed increased cartilage thickness and subchondral bone formation accompanied by narrowing of marrow cavities. Among the important findings were the reduction of disk thickness, the chondrocytes with normal architecture and some degenerative changes signs. (Fig.1, A1). Concomitantly Feizbakhsh et al., ${ }^{17}$ reported a significant higher cartilage thickness in rabbit's condyle due to HGH stimulatory effect.

The significant increase in cartilage and subchondral bone may be due to ability of HGH to stimulate mitosis, and to increase size and specific differentiation of cells such as bone forming cells. ${ }^{18}$ Livne et al., ${ }^{18}$ demonstrated that addition of $\mathrm{HGH}$ on mice chondrocytes may increase the anabolic activities of cell proliferation and sulfated proteoglycan synthesis. Lewinson et al., ${ }^{19}$ proved that HGH stimulates endochondral bone formation. In addition, the local administration of HGH may urge more specific effects and less systemic side effects. ${ }^{17}$

$\mathrm{H} \& \mathrm{E}$ stained sections of LLLTG showed chondrocytes with almost normal architecture and thickness, regenerated cartilage, and newly formed 
subchondral bone trabeculae with narrowing of the intervening marrow cavities. (Fig.1, A2). These findings, may be due to LLLT dose-specific, anti-inflammatory effect. ${ }^{20}$ Additionally LLLT up-regulates several cellular processes including oxidative phosphorylation that control biological function normalization at the cellular level. ${ }^{21}$ concomitantly Carvalho et al., ${ }^{8}$ and Lemos et al., ${ }^{15}$ demonstrated significant action of LLLT in treatment of arthritic rodent TMJ.

Disarrangement of collagen fibers with matrix defects or cavitation and loss of metachromasia were observed in CG (Fig.1, B\&C). These results were in agreement with Lemos et al.. ${ }^{15}$ who reported an increase loss of metachromasia and even loss of arrangement of collagen fiber and extracellular matrix (ECM) destruction in arthritic non-treated group..$^{22}$

Statistical analysis of values recorded from histomorphometrical analysis among the 3 groups revealed that, the HGHG showed a statistically significant difference when compared to the $\mathrm{CG}$ in all measurements except for lower compartment measurements. While when HGHG were compared to LLLTG, there was a statistically significant difference in all measurements except collagen average area, subchondral bone and upper compartment. Comparing the LLLTG with the CG there was a statistically significant difference in all measurements except disc thickness and upper compartment. The $\mathrm{P}$ value was $(\mathrm{P}=0.05)$. Table (1)

Regenerated collagen fibers with almost normal arrangement observed in the HGHG may be attributed to increase of collagen synthesis by $\mathrm{HGH} .{ }^{23}$ In addition the cartilage thickness increase with slight loss of the metachromatism were also reported. (Fig 1, B1\& C1). ${ }^{23}$ Similarly and in accordance with this study results (Fig 1, B2\& C2), Lemos et al., ${ }^{24}$ showed positive effects of LLLT via slight reduction in metachromasia and promoting arrangement of the collagen fibers. Additionally, Melis et al., ${ }^{25}$ revealed that LLLT not only increases the vascularization but also increases the fibroblast formation.

From the presented results both $\mathrm{HGH}$ and LLLT can accelerate the process of tissue repair and reduce the joint morphological changes. The superior regenerative effect of HGH over LLLT on

TABLE (1): Comparison between the three studied groups in relation to collagen average area, Marrow cavities percentage, cartilage thickness, subchondral bone trabeculae, upper and lower compartment and disk thickness using One Way ANOVA test followed by Tukey post hoc test.

\begin{tabular}{|c|c|c|c|c|}
\hline parameter & control & HGH & LLLT & P value \\
\hline Collagen Surface area & $3.2822^{\mathrm{A}} \pm .00763$ & $8.7428^{\mathrm{A}} \pm 1.71289$ & $5.7880^{\mathrm{A}} \pm 1.04612$ & $.001^{*}$ \\
\hline Cartilage & $50.4150^{\mathrm{A}} \pm 1.66923$ & $112.8430^{\mathrm{A}} \pm 28.049$ & $72.7280^{\mathrm{A}} \pm 6.43870$ & $.001 *$ \\
\hline Marrow cavities & $14.5330^{\mathrm{AB}} \pm 1.78791$ & $9.6680^{\mathrm{A}} \pm 1.26487$ & $10.9450^{\mathrm{B}} \pm 2.76587$ & $.001 *$ \\
\hline Lower compartment & $14.3770^{\mathrm{A}} \pm .93299$ & $12.5584^{\mathrm{AB}} \pm 0.49827$ & $15.6681^{\mathrm{B}} \pm 2.40464$ & $.001 *$ \\
\hline Upper compartment & $19.4170^{\mathrm{AB}} \pm 1.52259$ & $15.5420^{\mathrm{A}} \pm 2.0438$ & $16.5420^{\mathrm{B}} \pm 2.61201$ & $.001 *$ \\
\hline Disc thickness & $30.2002^{\mathrm{A}} \pm 1.76613$ & $62.9730^{\mathrm{A}} \pm 1.91515$ & $47.7794^{\mathrm{A}} \pm 5.61275$ & $.001 *$ \\
\hline Subchondral bone & $3.3100^{\mathrm{AB}} \pm 0.86111$ & $8.6500^{\mathrm{A}} \pm 1.76147$ & $9.1660^{\mathrm{B}} \pm 1.89454$ & $.001 *$ \\
\hline
\end{tabular}

Data were expressed as mean $\pm S D$

SD:standard deviation P:Probability

N.B: Same superscript letters in a row indicate significant difference $*(P<.05)$. 
the cartilage thickness might be due to the ability of $\mathrm{HGH}$, to stimulate progenitor cell proliferation, cartilage differentiation and mineralization of extracellular matrix, in addition to Growth factor1(IGF-1) local production which in turn stimulates bone and growth plate. ${ }^{26,27}$
Within the limitations of the current study, the results of HGH injection in guinea pigs' arthritic TMJ may successfully relieve many of RA features compared to LLLT. Further investigations are needed to study insulin growth factor (IGF) role before this step can be extrapolated to a human model.

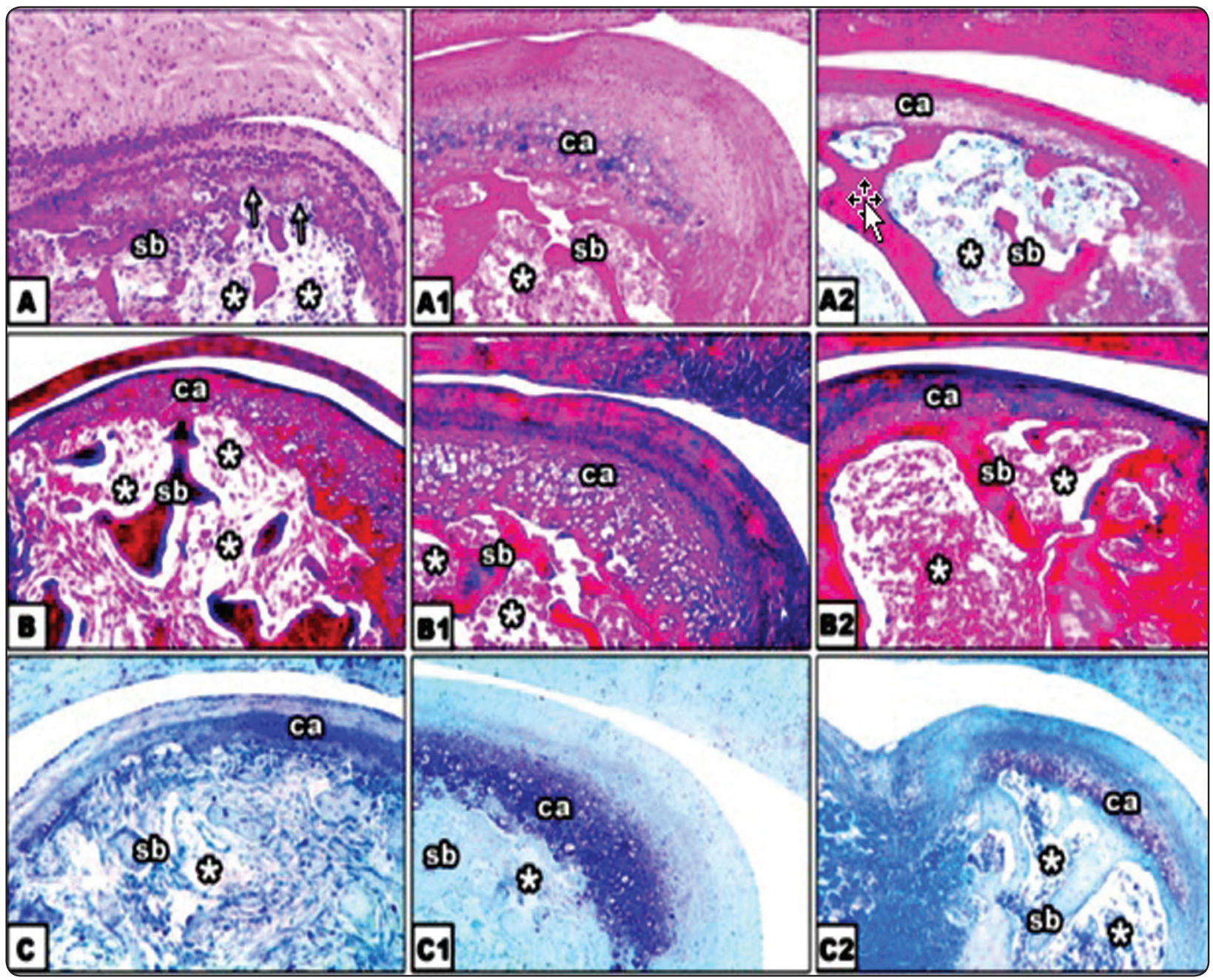

Fig. (1) : Coronal section of the TMJ in (A) control group showing thinning and irregularity of some parts of the articular cartilage, severe atrophy in other parts of the cartilage and destruction of the subchondral bone (sb). (A1) Coronal section of the TMJ in HGH group showing increase in the thickness of regenerated articular cartilage layer (ca). New regenerated subchondral bone trabeculae (sb) (H\&E X 100). (A2) Coronal section of the TMJ in LLLT group showing slight increase in the thickness of regenerated articular cartilage layer (sb) while decrease in marrow spaces (*) (H\&E X 100).(B) Coronal section of TMJ in control group showing marked decrease in articular cartilage (ca) thickness and disarrangement of collagen with matrix defects or cavitations. (Masson's Trichrome X100). (B1, B2) Coronal section of the condyle in HGH and LLLT group respectively showing regenerated collagen in some areas of regenerated cartilage (Masson's trichrome X 100). (C) Coronal section of TMJ in control group showed sever loss of the metachromatism in degenerated cartilage. (C1, C2) Coronal section of TMJ in HGH \& LLLT respectively showing slight loss of the metachromatism of both cartilage \& bone matrix. (Toluidine blue X 100). 


\section{REFERENCES}

1 Goater JJ, O'Keefe RJ, Rosier RN, et al. Efficacy of ex vivo OPG gene therapy in preventing wear debris induced osteolysis. J Orthop Res 2002;20:169-73.

2 Vincent TL, Williams RO, Maciewicz R, et al. Mapping pathogenesis of arthritis through small animal models. Rheumatology (Oxford) 2012;51:1931-41.

3 Padilla-Carlin DJ, McMurray DN, Hickey AJ. The guinea pig as a model of infectious diseases. Comp Med 2008;58:324-40

4 Villares R, Kakabadse D, Juarranz Y, et al. Growth hormone prevents the development of autoimmune diabetes. Proc Natl Acad Sci U S A 2013;110:4619-27.

5 Velloso CP. Regulation of muscle mass by growth hormone and IGF-I. Br J Pharmacol 2008;154:557-68.

6 Olney RC. Regulation of bone mass by growth hormone. Med Pediatr Oncol 2003;41:228-34.

7 Zhang JC, Lu HY, Lv GY, et al. The repair of critical-size defects with porous hydroxyapatite/polyamide nanocomposite: an experimental study in rabbit mandibles. Int J Oral Maxillofac Surg 2010;39:469-7.

8 Carvalho CM, Lacerda JA, dos Santos Neto FP, et al. Evaluation of laser phototherapy in the inflammatory process of the rat's TMJ induced by carrageenan. Photomed Laser Surg 2011;29:245-54.

9 Carlos FP, De Paula Alves Da Silva M, De Lemos Vasconcelos Silva Melo E, et al. Protective effect of low-level laser therapy (LLLT) on acute zymosan-induced arthritis. Lasers Med Sci 2014;29:757-3.

10 David L Scott, Frederick Wolfe TWJH. Rheumatoid arthritis. Lancet 2010;376:1094-08.

11 Takano A, Haruta T, Iwata M, et al. Growth hormone induces cellular insulin resistance by uncoupling phosphatidylinositol 3-kinase and its downstream signals in 3T3-L1 adipocytes. Diabetes 2001;50:1891-900.

12 Schurgers E, Billiau A, Matthys P. Collagen-Induced Arthritis as an Animal Model for Rheumatoid Arthritis: Focus on Interferon- $\gamma$. J Interferon Cytokine Res 2011;31:917-26.

13 Lin YC, Hsu ML, Yang JS, et al. Temporomandibular joint disorders in patients with rheumatoid arthritis. J Chin Med Assoc 2007;70:527-34.

14 Billiau a, Matthys P. Modes of action of Freund's adjuvants in experimental models of autoimmune diseases. J Leukoc Biol 2001;70:849-60.
15 Lemos GA, Rissi R, de Souza Pires IL, et al. Low-level laser therapy stimulates tissue repair and reduces the extracellular matrix degradation in rats with induced arthritis in the temporomandibular joint. Lasers Med Sci 2016;31:1051-9.

$16 \mathrm{Xu} \mathrm{L}$, Guo H, Li C, et al. A time-dependent degeneration manner of condyle in rat CFA-induced inflamed TMJ. Am J Transl Res 2016;8:556-67.

17 Feizbakhsh M, Razavi M, Minaian M, et al. The effect of local injection of the human growth hormone on the mandibular condyle growth in rabbit. Dent Res J 2014;11:2-7.

18 Livne E, Laufer D, Blumenfeld I. Comparison of in vitro response to growth hormone by chondrocytes from mandibular condyle cartilage of young and old mice. Calcif Tissue Int 1997;61:62-7.

19 Lewinson D, Bialik GADM. Effects of Hypothyroidism on the Cartilage and the Osteogenic Process in the Mandibular. Endocrinology 2015;135:1504-10.

20 Hashmi JT, Huang YY, Sharma SK, et al. Effect of pulsing in low-level light therapy. Lasers Surg Med 2010;42:460-6.

21 Herranz-Aparicio J, Vázquez-Delgado E, Arnabat-Domínguez J, et al. The use of low level laser therapy in the treatment of temporomandibular joint disorders. Review of the literature. Med Oral Patol Oral Cir Bucal 2013;18:603-12.

22 Wang Y, Zhao Y, Jia W, et al. Preliminary study on dental pulp stem cell-mediated pulp regeneration in canine immature permanent teeth. J Endod 2013;39:195-201.

23 Doessing S, Heinemeier KM, Holm L, et al. Growth hormone stimulates the collagen synthesis in human tendon and skeletal muscle without affecting myofibrillar protein synthesis. J Physiol 2010;588:341-51.

24 George Azevedo Lemos, Renato Rissi, Edson Rosa Pimentel ETP. Effects of high molecular weight hyaluronic acid on induced arthritis of the temporomandibular joint in rats. Acta Histochemica 2015;117:566-75.

25 Marcello Melis, Massimiliano Di Giosia KHZ. Low Level Laser Therapy for the Treatment of Temporomandibular Disorders: A Systematic Review of the Literature. Cranio 2012;30:304-12.

26 C. Pass, V.E. MacRae SFA and CF. Inflammatory cytokines and the GH/IGF-I axis: novel actions on bone growth. Cell Biochem Funct 2008;26:320-8.

27 Ramirez-Yañez GO, Young WG, Daley TJ, et al. Influence of growth hormone on the mandibular condylar cartilage of rats. Arch Oral Biol 2004;49:586-90. 